\title{
Atomic Force Microscopy Study of a Voltage Effect on CdZnTe Crystal Dimensions
}

\author{
M. Aleszkiewicz ${ }^{a, b}$, P. Aleszkiewicz ${ }^{a}$, T. Wojciechowski $^{a}$, \\ K. Fronc ${ }^{a}, \mathrm{~V} . \mathrm{Kolkovsky}^{a}$, E. JAniK ${ }^{a}$, R. JakięA ${ }^{a}$, \\ A. MYCIELSKI ${ }^{a}$ AND G. KARCZEWSKI ${ }^{a}$ \\ ${ }^{a}$ Institute of Physics, Polish Academy of Sciences \\ al. Lotników 32/46, 02-668 Warsaw, Poland \\ ${ }^{b}$ Dept. Mathematics and Natural Sciences, College of Sciences \\ UKSW, Warsaw, Poland
}

\begin{abstract}
In this work we studied the influence of an external electric voltage on spatial dimensions of CdZnTe mixed crystals. In order to get an absolute magnitude of the sample thickness and to gain insight to the changes of lateral dimension, in quasi-bulk $3 \mu \mathrm{m}$ thick CdZnTe layers grown by molecular beam epitaxy square craters were formed by ion sputtering in a secondary ion mass spectrometer. The vertical and lateral dimensions of the craters were studied by the atomic force microscopy. The atomic force microscopy measurement revealed that the thickness of the CdZnTe layer increases in a result of applying a single voltage pulse to the sample surface and decreases reversibly after applying reversely biased voltage. The voltage triggering was high enough to switch the conductivity state of the sample i.e., the effect of thickness change is accompanied by the effect of conductivity switching. The thickness change is significant, reaching several percents of the entire layer thickness.
\end{abstract}

PACS numbers: 73.40.Ns, 73.61.Ga, 77.90.+k

\section{Introduction}

Since the discovery of ferroelectricity in CdZnTe crystals in 1989 [1], the material draws attention as applicable in nonvolatile memories $[2,3]$. In comparison to classical ferroelectrics - for instance, perovskites [4] — the II-VI mixed crystals are semiconductors and carrier effects must be taken into account. The importance of carriers is affirmed by reversible conductivity switching exhibited by CdZnTe bulk samples and epitaxial films whereas the resistivity can be changed 
by a few orders of magnitude by applying large external voltage [5, 6]. Except of the presence of carriers there is a more serious problem which makes the existence of ferroelectric phase in CdZnTe crystals questionable. Namely, ferroelectricity is forbidden in high symmetry (cubic) crystals. However, in spite of an extensive experimental search, no lattice distortion from the zinc blende cubic structure was found in CdZnTe with low Zn content. On the other hand, there are predictions that off-site ion occupancy may cause a local lattice distortion and thus may lead to ferroelectricity [7]. CdZnTe is a very good candidate to fulfill this condition because $\mathrm{Zn}$ ions are $20 \%$ smaller than Cd ions. Because of the ionic radius difference, small $\mathrm{Zn}$ atoms are likely to move from substitutional positions in cubic lattice to interstitial positions with tetragonal symmetry. This process leads to lowering of the local crystal symmetry and to formation of a $\mathrm{Cd}$ vacancy-interstitial $\mathrm{Zn}$ dipole pair which may be the origin of the electric polarization observed in the CdZnTe crystals [8].

Since by direct experimental methods as X-ray diffraction (XRD) no lattice distortion in CdZnTe has been found yet, here we report on indirect evidences of the effect. The aim of this work is to show that an external voltage applied to a CdZnTe sample results not only in conductivity switching but also in reversible changes of the sample dimensions. The spatial dimension changes were detected by atomic force microscope (AFM) which offers possibility of precise, sub-nanometer distance measurements both in lateral and vertical direction.

\section{Experimental}

In the preliminary experiment continuous change of sample dimensions by biasing with continuous ac voltage was measured i.e., the sample thickness measurement took place upon the voltage application. The sample was an about $0.5 \mathrm{~mm}$ thick $\mathrm{Cd}_{0.96} \mathrm{Zn}_{0.04}$ Te plate with (211) oriented surface cut from a bulk crystal grown by the Bridgman method. It was checked that it does show hysteretic conductivity switching between $0.3 \mathrm{M} \Omega$ and $4 \mathrm{M} \Omega$ as a result of applying several hundreds of volts (the larger the sample dimensions the higher voltage is necessary for switching). The reference CdTe sample was about $1 \mathrm{~mm}$ thick plate with (111) surface. Au contacts were sputtered on both - upper and bottom - sides of the CdZnTe and CdTe crystals and the contacting wires were connected. The samples were excited with sinusoidal alternating (ac) voltage from a generator (with maximum peak-to-peak value $10 \mathrm{~V}$, i.e., well below the switching value). Thin slice of mica was glued on the top of the samples to ensure atomically flat surface beneath the AFM tip. The vertical elongation of a sample as a response to applied external voltage was detected in AFMicroscope (MultiMode Nanoscope IIIA, Digital Instruments) in tapping mode using $100 \mathrm{kN} / \mathrm{m}$ silicon nitride tips. The $X Y$ (lateral) scanning was disabled therefore the image lines show temporal change in vertical tip displacement. In the main experiment sample dimensions were measured before and after switching of the conductivity state by 


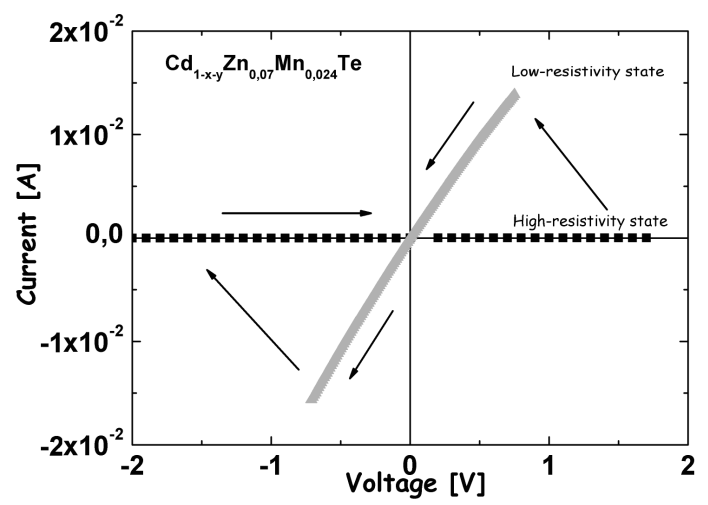

Fig. 1. Reversible hysteretic switching of $3 \mu \mathrm{m}$ thick $\mathrm{Cd}_{1-x-y} \mathrm{Zn}_{0.07} \mathrm{Mn}_{0.024} \mathrm{Te}$.

a single voltage pulse. The sample was quasi-bulk - approximately $3 \mu \mathrm{m}$ thick - $\mathrm{Cd}_{0.906} \mathrm{Zn}_{0.07} \mathrm{Mn}_{0.024}$ Te layer grown by MBE on (100) oriented $n$-type GaAs. It showed good reproducibility in hysteretic switching (Fig. 1) between two conductivity states of $10 \Omega$ and $10 \mathrm{k} \Omega$ for the particular sample used. We believe the influence of low content of manganese (whose ionic radius is only slightly smaller than that of cadmium) is negligible for symmetry considerations - at least in comparison with the role of relatively high $\mathrm{Zn}$ content in the sample used in experiment. Square holes (craters) of different depths and diameters were fabricated in the layer by ion sputtering in secondary ion mass spectroscope (SIMS) using oxygen ion beam. The deepest crater preparation was terminated after the GaAs substrate was reached. The crater dimensions were optimized for AFM imaging (Nanoscope IIIA) with scanning area $X Y<200 \mu \mathrm{m}$ and vertical range $<5.8 \mu \mathrm{m}$.

\section{Results and discussion}

\subsection{Extension caused by external voltage}

The aim of the first, preliminary experiment was to examine and estimate the piezoelectric properties of CdZnTe. From symmetry considerations it is known that all ferroelectric crystals are also piezoelectric (i.e., a voltage is generated on piezoelectric sample surfaces in response to an elastic strain and inversely - a change of sample dimensions occurs upon biasing the sample with an external voltage). Bulk CdZnTe sample shows sinusoidal vertical elongation when biased with the sinusoidal external voltage applied over the sample thickness. The elongation of the sample is approximately $1 \mathrm{~nm}$ for $1 \mathrm{~V}$ pp voltage. Unfortunately, the reference bulk CdTe sample exhibits a very similar effect where external sinusoidal voltage results in the vertical elongation of $1 \mathrm{~nm}$ per $1 \mathrm{~V} \mathrm{pp}$, too. The CdTe crystallizes in zinc blende structure and is not ferroelectric, so the observed effect in $\mathrm{CdTe}$ cannot be of the piezoelectric origin. The elongation may be assigned to the heating by the current flowing through the sample. Let us note that the sample 
thicknesses of CdZnTe and CdTe are about $0.5 \mathrm{~mm}$ and $1 \mathrm{~mm}$, respectively, so the net elongation is larger for CdZnTe than for CdTe. The proper adjustment of voltage frequency should enable avoiding thermal expansion - when voltage changes are fast enough, thermal expansion caused by current is excluded because of thermal inertia. However, the maximum AFM scan rate of $20 \mathrm{~Hz}$ (40 lines per s) limits the voltage frequencies which can be applied to about $200 \mathrm{~Hz}$ (then 2 voltage periods pass per scan line). Moreover, the scan pattern (trace + retrace + scan direction reversal) excludes the possibility of phase determination. Even for relatively high voltage frequency of $200 \mathrm{~Hz}$ the extension (of non-ferrroelectric origin) in $\mathrm{CdTe}$ is still visible. It can be concluded that the vertical elongations for CdTe and CdZnTe of comparable thicknesses are comparable. For both measured samples the elongation per voltage is the same, but the sample thickness is about twice higher for CdTe, which means that the elongation per sample thickness is smaller in this case. However, it cannot be directly subtracted as non-ferroelectric part from the elongation curves for CdZnTe because of the differences in heat conductance of CdTe and CdZnTe. Therefore in order to measure the effect of the electric field on the dimensions of the samples the experiment has to be carried out in the absence of the current flow.

\subsection{Dimension change caused by switching}

The reversible switching between two resistivity states of CdZnTe is believed to be caused by the movement of zinc atoms in the crystal lattice between substitutional and interstitial positions. Since the zinc content is of the order of a few percents, the change of the sample thickness which may accompany this movement is expected to be quite small. The sample thickness measurements were performed on a quasi-bulk CdZnTe MBE grown epitaxial layer with $7 \%$ Zn content. The idea is to measure lateral and vertical dimensions change of the layer as the change in diameter and depth of a SIMS sputtered crater in both conductivity states of the sample. For that reason AFM images of the same surface region before and after conductivity switching by an electrical pulse were compared. The large-diameter craters of depths of $2.5 \mu \mathrm{m}$ and $<1 \mu \mathrm{m}$ in as-grown (high resistivity) state were measured and their diameter (measured at half of the depth) was $150 \mu \mathrm{m}$. The sample was then switched ex situ (i.e., outside of the AFMicroscope) to the low resistivity state and the same crater area was measured again with AFM. In order to achieve the conductivity switch the dc voltage is swept until the conductivity fell to its lower value which occurred at about $20 \mathrm{~V}$ (in the lateral - in-plane direction). In a result, the crater diameter is remarkably decreased. The change of the $150 \mu \mathrm{m}$ wide crater diameter is of about $10 \mu \mathrm{m}$ as shown in Fig. 2 . In this case the change of the crater height cannot be determined as the crater walls are on the $X Y$ scan limits of the AFM. In the case of the shallow crater (depth $<1 \mu \mathrm{m}$ ) it was not possible to make a precise comparison of the diameter before and after switching since the conductivity switching was done ex situ and the difference in sample tilt was larger than crater depth. The effect of crater diameter decrease 
seems to be caused by lateral expansion of the sample rather than by vertical, since the amount of the CdZnTe material to be expanded vertically in the vicinity of the crater bottom is negligible.

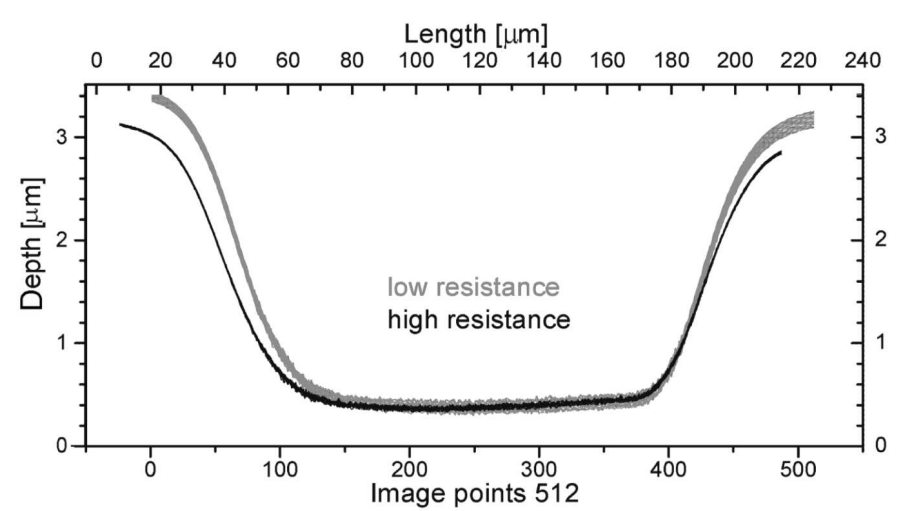

Fig. 2. AFM scan lines of an ion sputtered crater: the half height diameter $150 \mu \mathrm{m}$ is decreased by about $10 \mu \mathrm{m}$ after switching the sample from high to low resistivity state. For low-resistivity profiles the sample tilt had to be subtracted (line if 1st order) because the switching was realized ex situ; therefore the simultaneous increase in the crater depth — as situated on lateral AFM scanning limits — may be misleading.

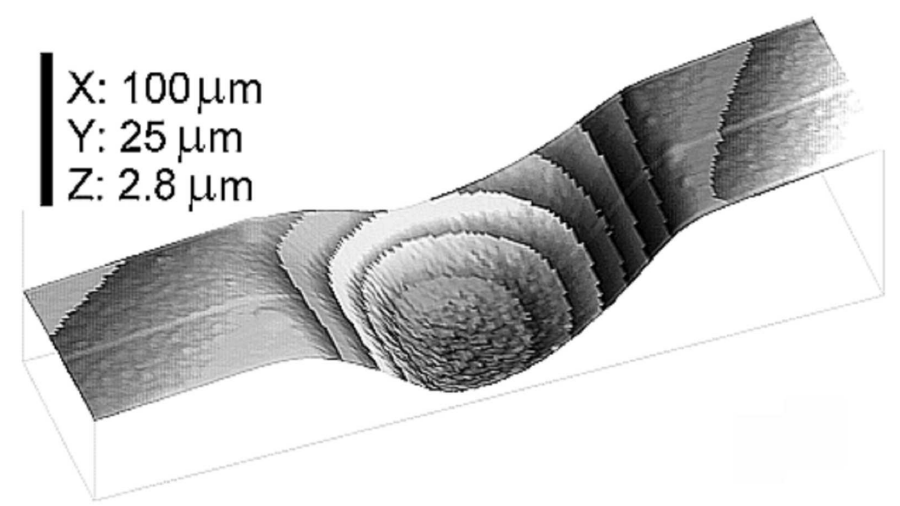

Fig. 3. AFM image of a $30 \mu \mathrm{m}$ diameter ion sputtered crater. Since the bottom is rounded, the depth must be checked on precisely defined localizations.

Since the switching is reversible, the reproducibility of the thickness change can be checked. For the measurement of sample thickness difference in two conductivity states several craters of smaller diameters of about $30 \mu \mathrm{m}$ were prepared by SIMS (Fig. 3). The bottom of the deepest crater reaches GaAs substrate and other craters are slightly shallower but deep enough to give measurable layer thickness change. The contacts to the sample were fabricated small enough to enable the 


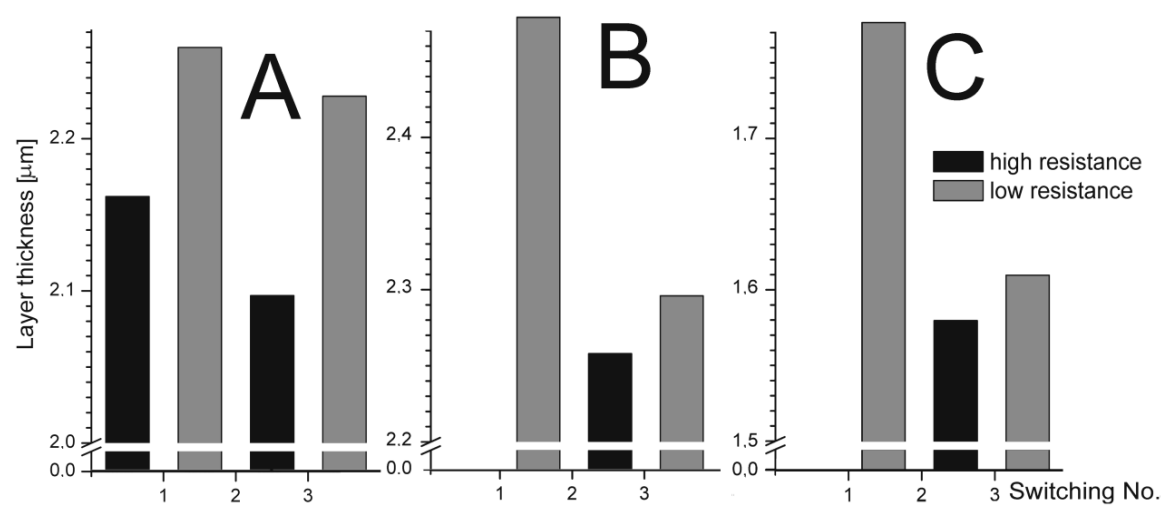

Fig. 4. Comparison of crater depths for three different craters A, B, C after 3 consecutive switches (first switch from as-grown high resistivity state to low resistivity). There is a clear correspondence between low resistivity state and increased thickness or inversely. The absolute depth of craters in consecutive states of the same conductivity differs slightly; some part of this difference is caused by non-zero surface roughness (CdZnTe root mean square is about $10 \mathrm{~nm}$ on $20 \mu \mathrm{m}$ surface length).

conductivity switching in situ i.e., without taking the sample out of the AFMicroscope. It was found that the switching from the as-grown (high resistivity) state to the low resistivity state results in increase in the layer thickness (crater depth). As in the case of the wide-diameter crater the change is again significant, about $100 \mathrm{~nm}$ elongation for the $2 \mu \mathrm{m}$ total depth of the crater. When the sample is switched back to high resistivity state (by an oppositely biased voltage), the sample thickness decreases to the initial value. Since the bottom of the craters is not flat, special care must be taken for choosing exactly the same scan lines for comparison. This was done by seeking for surface imperfections as markers. Craters of different depths show the same correspondence of low resistivity - high volume and inversely. After consecutive conductivity switches the depth of three craters was checked. In all cases the crater depth (i.e. layer thickness) was higher for low resistivity state (Fig. 4).

\section{Conclusions}

The AFM scan rate used to detect temporal changes of sample dimension caused by external voltage is limited. It was expected that it would be possible to find parameters (voltage frequency, scan rate) which for bulk CdTe gave no elongation. Since the thermal properties of zinc blende structure of CdTe cannot be straightforwardly compared to the lower symmetry structure of CdZnTe it was not possible to extract purely piezoelectric part of thickness change. The CdZnTe layer dimensions in high and low resistivity state was compared. The diameters and depths of craters sputtered in approximately $2 \mu \mathrm{m}$ thick layer were measured resulting in remarkably high change in layer dimensions. It can be concluded that 
the low resistivity state has higher lattice constant but the several \% of dimension change must be taken with caution. Behavior of the hole structure (the outer part of which has no lattice surrounding) in the material may differ substantially from that of the continuous layer.

\section{Acknowledgments}

This research was partially supported by the Ministry of Science and Higher Education (Poland) through the grant N20205432/1189.

\section{References}

[1] R. Weil, R. Nkum, E. Muranevich, L. Benguigui, Phys. Rev. Lett. 62, 2744 (1989).

[2] D.J. Fu, J.C. Lee, S.W. Choi, S.J. Lee, T.W. Kang, Appl. Phys. Lett. 81, 5207 (2002).

[3] D.J. Fu, J.C. Lee, W.C. Lee, D.H. Kim, D.J. Lee, T.W. Kang, J. Korean Phys. Soc. 45, Suppl. S, S633 (2004).

[4] A. Beck, J.G. Bednorz, Ch. Gerber, C. Rossel, D. Widmer, Appl. Phys. Lett. 77, 139 (2000).

[5] T. Wojciechowski, E. Janik, E. Dynowska, K. Fronc, G. Karczewski, Phys. Status Solidi C 3, 1197 (2006).

[6] T. Wojciechowski, P. Jakubas, P. Boguslawski, G. Karczewski, J. Korean Phys. Soc. 53, 23 (2008).

[7] Q. Islam, B. Bunker, Phys. Rev. Lett. 59, 2701 (1987).

[8] P. Jakubas, P. Boguslawski, Phys. Rev. B 77, 214104 (2008). 